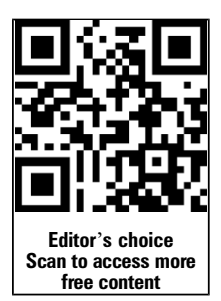

${ }^{1}$ Division of Physiotherapy, Department of Medical and Health Sciences, Linköping University, Linköping, Sweden ${ }^{2}$ Football Research Group, Linköping University, Linköping, Sweden ${ }^{3}$ Division of Community Medicine, Department of Medical and Health Sciences, Linköping University, Linköping, Sweden

\section{Correspondence to} Martin Hägglund, Division of Physiotherapy, Department of Medical and Health Sciences, Linköping University, Linköping 581 83, Sweden; martin.hagglund@liu.se

Accepted 4 April 2013 Published Online First 3 May 2013
To cite: Hägglund $M$, Waldén $M$, Magnusson $H$, et al. Br J Sports Med 2013:47:738-742.

\title{
Injuries affect team performance negatively in professional football: an 11-year follow-up of the UEFA Champions League injury study
}

\author{
Martin Hägglund, ${ }^{1,2}$ Markus Waldén, ${ }^{2,3}$ Henrik Magnusson, ${ }^{1,2}$ Karolina Kristenson, 2,3 \\ Håkan Bengtsson, ${ }^{2}$ Jan Ekstrand ${ }^{2,3}$
}

\begin{abstract}
Background The influence of injuries on team performance in football has only been scarcely investigated.
\end{abstract}

Aim To study the association between injury rates and team performance in the domestic league play, and in European cups, in male professional football.

Methods 24 football teams from nine European countries were followed prospectively for 11 seasons (2001-2012), including 155 team-seasons. Individual training and match exposure and time-loss injuries were registered. To analyse the effect of injury rates on performance, a Generalised Estimating Equation was used to fit a linear regression on team-level data. Each team's season injury rate and performance were evaluated using its own preceding season data for comparison in the analyses.

Results 7792 injuries were reported during 1026104 exposure hours. The total injury incidence was 7.7 injuries/ $1000 \mathrm{~h}$, injury burden 130 injury days lost/1000 h and player match availability $86 \%$. Lower injury burden $(p=0.011)$ and higher match availability $(p=0.031)$ were associated with higher final league ranking. Similarly, lower injury incidence $(p=0.035)$, lower injury burden $(p<0.001)$ and higher match availability $(p<0.001)$ were associated with increased points per league match. Finally, lower injury burden ( $p=0.043)$ and higher match availability $(p=0.048)$ were associated with an increase in the Union of European Football Association (UEFA) Season Club Coefficient, reflecting success in the UEFA Champions League or Europa League.

Conclusions Injuries had a significant influence on performance in the league play and in European cups in male professional football. The findings stress the importance of injury prevention to increase a team's chances of success.

\section{INTRODUCTION}

Injuries are common in international and professional football, and injuries are the most common reason for player unavailability in training and matches. ${ }^{1-3}$ Injuries during a season or a tournament could thus have a considerable impact on a team's performance.

In one study of the Swedish men's national team over 6 years, injury incidence was higher during matches lost or tied compared with matches won. ${ }^{4}$ This finding was not repeated, however, in a study on nine men's (senior, under-21, under-19 and under-17) and three women's (under-19) European Championships between 2006 and 2008, where no difference in injury incidence was seen based on match results. ${ }^{5}$
In the women's European Championship in 2005, teams that were eliminated in the group stage of the tournament had a higher match injury incidence than teams that advanced to the semifinals, while no such association was found in the men's tournament 2004 or in the men's under-19 tournament in 2005 . $^{6}$ No difference in total injury incidence between teams that were eliminated in the group stage and those that qualified for the final stage of the tournament was reported in the previously mentioned study on 12 European Championships. ${ }^{5}$

In club level football, three studies have correlated injury figures with team performance in the league play. In one study including 17 teams in the Icelandic top two male divisions during one season, a relationship, although non-significant $(p=0.092)$, between the number of injury days per team and the final league ranking was found, indicating that teams with fewer injury days had a better chance of success. ${ }^{7}$ In another study on a French professional team followed over 15 seasons, no correlation between the team's final league ranking and total injury incidence in a season was observed. ${ }^{8}$ Finally, a study on the Qatari professional league reported strong correlations between injury incidence and a high league ranking, more games won, more goals scored, greater goal difference and total number of points in a season. ${ }^{9}$

Despite some contradictory findings, studies indicate that injuries may have an impact on a team's performance. To the authors' knowledge, the association between injury rates and team performance in the European cups administered by the Union of European Football Associations (UEFA) has not been studied previously. Therefore, the objective of this study was to investigate the association between injury rates and performance in the domestic league play and in European cups in male professional football. Our hypothesis was that there would be an association between lower injury rates and improved team performance in both domestic and international competitions.

\section{MATERIALS AND METHODS}

The so-called UEFA Champions League (UCL) injury study is an ongoing prospective injury surveillance study of male professional football that started in 2001. ${ }^{10}$ The total study cohort includes 27 teams invited by UEFA with the criteria that they had participated at the highest level in Europe over the last decade. ${ }^{11}$ The present study was a subanalysis of the UCL injury study where only teams with at least two consecutive seasons of data delivery between 2001 and 2012 were included. This yielded 24 teams from 
nine European countries (table 1), with a total of 155 teamseasons (out of 160 in the total study cohort; 3 excluded teams participated in 1 season each, 2 included teams suffered points deduction in the league play in the 2005/2006 season and these 2 team-seasons were also excluded). All teams participated in the highest domestic competition and also regularly in the UCL or Europa League (EL) tournaments.

\section{Data collection}

Data collection procedures and definitions followed the UEFA guidelines, ${ }^{12}$ and harmonised with the consensus statement for football injury surveillance. ${ }^{13}$ Individual player participation in training and matches was registered by a contact person in each team on an exposure form and sent to the study group on a monthly basis. All team training and match exposures were included. The first team medical staffs recorded injuries on an injury form that was sent to the study group each month. The injury form had information about the diagnosis, nature and circumstances of injury occurrence. A recordable injury was defined as any physical complaint sustained by a player that resulted from a football match or football training and led to the player being unable to take a full part in future football training or match play (ie, time-loss injury). The player was considered injured until the medical staff allowed full participation in training and availability for match selection. Injury absence was measured as the number of days from injury occurrence to full participation.

\section{Evaluation of team season performance and injury rates}

Team season performance was evaluated using three different measures: the UEFA Season Club Coefficient (UEFA SCC), final league ranking and points per league match. UEFA SCC was included to represent a team's international performance in the European cups. The coefficient is based on the results of teams competing in the UCL and EL (including former UEFA Cup) tournaments. Teams are awarded points based on how far they reach in the tournaments, and the result in group stage matches. UEFA SCC is determined by the sum of all points won in the current season, plus $20 \%$ of the national association coefficient over the same period (the association coefficient takes into account the results of all teams from each association). ${ }^{14}$ Final league ranking and points per league match ('total league points/league matches played') were used to represent a team's domestic league performance.

Team injury rates during the season were also evaluated in three different ways: injury incidence, injury burden and match availability. Injury incidence was expressed as the number of injuries per $1000 \mathrm{~h}$ of football training and match play. Injury burden was calculated as the number of injury days lost per $1000 \mathrm{~h}$ ('injury incidence $\times$ mean absence per injury'), thus accounting for both the frequency and severity of injuries. Player match availability was calculated as ' $\Sigma$ of player match opportunities (=number of team matches $\times$ squad size) $-\Sigma$ of player match absences due to injury or illness', and expressed as the average season match availability in $\%$. Availability was calculated separately for league matches and European cup matches.

\section{Statistical analyses}

Since teams from nine leagues with different competition formats were included, each team's season performance measure was evaluated using its own preceding season performance for comparison. The change in performance between seasons was calculated as 'performance in season 2-performance

Table 1 Inclusion of the 24 teams over the 11 -year study period

\begin{tabular}{|c|c|c|c|c|c|c|c|c|c|c|c|c|c|}
\hline Team & Country & $01 / 02$ & $02 / 03$ & 03/04 & $04 / 05$ & $05 / 06$ & $06 / 07$ & $07 / 08$ & $08 / 09$ & $09 / 10$ & $10 / 11$ & $11 / 12$ & Total \\
\hline Club Brügge KV & Belgium & & & $X^{*}$ & $x$ & $x$ & $x$ & $x$ & $x$ & $x$ & $x$ & $x$ & 9 \\
\hline RSC Anderlecht & Belgium & & & & & & $X^{*}$ & $x$ & $x$ & & & $X^{*}$ & 4 \\
\hline Arsenal FC & England & $X^{*}$ & $x$ & $x$ & $x$ & $x$ & $x$ & $x$ & $x$ & $x$ & $x$ & $x$ & 11 \\
\hline Chelsea FC & England & & & & & $X^{*}$ & $x$ & $x$ & & & $X^{*}$ & $x$ & 5 \\
\hline Liverpool FC & England & & & & & $X^{*}$ & $x$ & & & $X^{*}$ & $x$ & $x$ & 5 \\
\hline Manchester United FC & England & $X^{*}$ & $x$ & $x$ & $x$ & $x$ & $x$ & $x$ & & & $X^{*}$ & $x$ & 9 \\
\hline Tottenham Hotspur FC & England & & & & & & & & & & $\mathrm{X}^{*}$ & $x$ & 2 \\
\hline Olympique Lyonnais & France & & & & & & & & $X^{*}$ & $x$ & $x$ & $x$ & 4 \\
\hline RC Lens & France & $\mathrm{X}^{*}$ & $x$ & $x$ & & & & & & & & & 3 \\
\hline Paris St Germain & France & $X^{*}$ & $x$ & $x$ & $x$ & $x$ & $x$ & & & & & & 6 \\
\hline Stade Rennais FC & France & $X^{*}$ & $x$ & & & & & & & & & & 2 \\
\hline BVB Dortmund & Germany & & & & & $X^{*}$ & & & & $X^{*}$ & $x$ & $x$ & 4 \\
\hline FC Bayern München & Germany & & & & & & & & & $X^{*}$ & $x$ & $x$ & 3 \\
\hline Hamburger SV & Germany & & & & & & $X^{*}$ & $x$ & $x$ & $x$ & $x$ & & 5 \\
\hline AC Milan & Italy & $X^{*}$ & $x$ & $x$ & & & $X^{*}$ & & & $X^{*}$ & $x$ & $x$ & 7 \\
\hline FC Internazionale & Italy & $\mathrm{X}^{*}$ & $x$ & $x$ & $x$ & $x$ & $x$ & $x$ & $x$ & $x$ & $x$ & $x$ & 11 \\
\hline Juventus FC & Italy & $X^{*}$ & & $X^{*}$ & $x$ & & & & $X^{*}$ & $x$ & $x$ & $x$ & 7 \\
\hline AFC Ajax & The Netherlands & $\mathrm{X}^{*}$ & & & & $X^{*}$ & $x$ & $x$ & $x$ & $x$ & $x$ & $x$ & 8 \\
\hline PSV Eindhoven & The Netherlands & $X^{*}$ & $x$ & $x$ & $x$ & $x$ & $x$ & $x$ & $x$ & $x$ & $x$ & $x$ & 11 \\
\hline FC Porto & Portugal & & & & & $\mathrm{X}^{*}$ & $x$ & $x$ & $x$ & $x$ & $x$ & $x$ & 7 \\
\hline SL Benfica & Portugal & & & & & $\mathrm{X}^{*}$ & $x$ & $x$ & $x$ & $x$ & $x$ & $x$ & 7 \\
\hline FC Barcelona & Spain & & & $X^{*}$ & $x$ & $x$ & $x$ & $x$ & $x$ & $x$ & $x$ & $x$ & 9 \\
\hline Real Madrid CF & Spain & $X^{*}$ & $x$ & $x$ & $x$ & $x$ & $x$ & $x$ & $x$ & $x$ & $x$ & $x$ & 11 \\
\hline FC Shakhtar & Ukraine & & & & & & & $X^{*}$ & $x$ & $x$ & $x$ & $x$ & 5 \\
\hline Total & & 11 & 9 & 11 & 9 & 14 & 16 & 14 & 14 & 17 & 20 & 20 & 155 \\
\hline
\end{tabular}

*Thirty-four team-seasons were excluded from the analysis because they were not preceded by an adjacent season in the study (index season in study $\mathrm{n}=24$, partial discontinuation of study inclusion $n=10$ ). 
in season 1'. Similarly, injury rates vary between different teams (table 2), and to adjust for these variations, each team's season injury rate was also evaluated using its own preceding season injury rate for comparison. The change in injury rate between seasons was calculated as 'injury rate in season 2-injury rate in season 1'.

To be able to calculate between-season changes in injury rate and performance, only seasons that were preceded by a season of data delivery in the study were included $(n=121)$. Thirty-four team-seasons were excluded from the analysis because they were not preceded by an adjacent season in the study (index season in study $n=24$, partial discontinuation of study inclusion $n=10$ ). In the analyses of UEFA SCC, only seasons with at least two consecutive seasons of participation in the UCL or EL tournaments were included $(n=111)$.

To analyse the effect of injury rates on performance, a Generalised Estimating Equation with identity link was used to fit a linear regression on team level data, with each team-season change as an observation. To account for correlated observations, team was treated as a repeated measure. The SPSS procedure GENLIN (link=identity, distribution=normal, working correlation structure $=$ independent) was used. As an indication of the model goodness of fit, the Corrected Quasi likelihood under Independence model Criterion (QICC) was used, and the independent working correlation structure generated the lowest QICC in all models. The effects from the different injury rate measures (change in injury incidence, injury burden and match availability) on the different performance measures (change in UEFA SCC, league ranking and points per league match) were analysed in separate models. Both the crude and adjusted analyses were carried out. Adjustment was made for the variable 'change of head coach' before the analysed season (dichotomous variable: new coach or same coach). In addition, analyses of the variables league ranking and points per league match were

Table 2 Player availability, injuries and team performance

\begin{tabular}{|c|c|c|c|c|}
\hline & Mean & SD & Median & Range \\
\hline \multicolumn{5}{|l|}{ Player availability (\%) } \\
\hline Training & 77.0 & 6.1 & 77.3 & $61.6-91.1$ \\
\hline Match total & 86.0 & 4.7 & 85.9 & $74.5-95.4$ \\
\hline League match & 86.6 & 5.1 & 86.5 & $74.8-96.4$ \\
\hline UCL or EL match & 86.7 & 5.1 & 87.3 & $75.8-95.8$ \\
\hline \multicolumn{5}{|l|}{ Injuries } \\
\hline \multicolumn{5}{|l|}{ Number of injuries/team/season* } \\
\hline Total & 52.0 & 21.2 & 52 & $12-121$ \\
\hline Training & 22.7 & 11.8 & 21 & $2-58$ \\
\hline Match play & 29.3 & 12.5 & 29 & $6-63$ \\
\hline \multicolumn{5}{|l|}{ Injury incidence per $1000 \mathrm{~h}$} \\
\hline Total & 7.7 & 3.3 & 7.5 & $1.7-20.5$ \\
\hline Training & 4.0 & 2.1 & 3.6 & $0.5-12.1$ \\
\hline Match play & 26.6 & 10.7 & 26.6 & $6.7-57.0$ \\
\hline Injury burdent & 130.0 & 52.7 & 135.6 & $15.6-238.5$ \\
\hline \multicolumn{5}{|l|}{ Team performance } \\
\hline Final league ranking & 3.0 & 2.6 & 2 & $1-15$ \\
\hline Points per league match & 2.0 & 0.3 & 2.0 & $1.1-2.8$ \\
\hline UEFA Season club coefficient & 17.8 & 8.9 & 17.6 & $0-38.4$ \\
\hline
\end{tabular}

*Value was approximated to an 11-month season in case a partial season was included (ie, number of training sessions reported/number of months of data reporting $\times 11$ ).

tInjury days lost/1000 $\mathrm{h}$ (injury incidencexmean absence per injury).

EL, Europa League; UCL, UEFA Champions League; UEFA, Union of European Football

Associations. adjusted for the variable UEFA SCC, and vice versa, analyses of UEFA SCC were adjusted for the variable points per league match, to account for the possible correlation between a team's season performance in the domestic league and European cups.

All analyses were two-sided and the significance level set at $\mathrm{p}<0.05$.

\section{RESULTS}

In total, 7792 injuries (3395 training, 4397 match play) during $1026104 \mathrm{~h}$ of football training and match play were included.

\section{Availability and injury rates}

The mean player availability was $77 \%$ in training and $86 \%$ at matches (table 2). The mean injury incidence was 7.7 injuries/ $1000 \mathrm{~h}$ of total exposure, and injury burden 130 days lost/ $1000 \mathrm{~h}$. There was substantial variation in player availability and injury rates between teams and seasons (table 2).

\section{Team performance}

The median number of points per league match was 2.0 and the median final league ranking was second place (table 2). In 51 team-seasons (33\%), the ranking was first place, in 36 seasons (23\%) second place, in 29 seasons (19\%) third place, in 16 seasons $(10 \%)$ fourth place and in the remaining 23 seasons $(15 \%)$, the team finished in the fifth place or lower in the final league ranking. The median UEFA SCC was 17.6 points, and teams were in the top 20 ranking of European teams in 80 of 155 team-seasons (52\%).

\section{Injury rates and performance}

Injury rates showed several effects on team performance in the domestic league as well as in European cups (table 3). The results of adjusted analyses showed that lower injury burden $(p=0.011)$ and higher match availability $(p=0.031)$, compared with the preceding season, were associated with a higher final league ranking. Similarly, lower injury incidence $(p=0.035)$, lower injury burden $(\mathrm{p}<0.001)$ and higher match availability $(p<0.001)$ were associated with increased average points per league match. Finally, lower injury burden $(p=0.043)$ and higher match availability $(\mathrm{p}=0.048)$ were associated with an increase in UEFA SCC.

\section{DISCUSSION}

The main finding was that there were significant associations between low season injury rates and increased performance in the domestic league as well as in European cups for professional football teams. These were all prominent European teams, finishing in the top three positions in their domestic leagues in $75 \%$ of the included seasons, and in the top 20 European club ranking in 52\% of the seasons. The association between injuries and performance is probably one of the most important messages to convey to technical and coaching staff, as well as to other stakeholders in professional sports clubs, in order to continue to improve medical services for the players and to increase efforts to prevent injuries.

\section{Injury rates and team performance}

Injury burden and match availability showed effects on all three performance measures according to the adjusted analyses, while injury incidence was associated only with points per league match. This means that a team that had both decreased injury rates and injury severity compared with the preceding season had a statistically better chance of improved team performance. 
Table 3 Association between team season injury rates and performance in professional football

\begin{tabular}{|c|c|c|c|c|c|c|}
\hline & \multicolumn{3}{|c|}{ Crude analyses* } & \multicolumn{3}{|c|}{ Adjusted analyses* } \\
\hline & $\boldsymbol{\beta}$ & $95 \% \mathrm{Cl}$ & p Value & $\boldsymbol{\beta}$ & $95 \% \mathrm{Cl}$ & $\mathrm{p}$ Value \\
\hline \multicolumn{7}{|l|}{ Final league rankingt } \\
\hline Injury incidence & -0.057 & -0.187 to 0.072 & 0.386 & -0.049 & -0.161 to 0.062 & 0.387 \\
\hline Injury burden & -0.009 & -0.017 to -0.002 & 0.015 & -0.010 & -0.017 to -0.002 & 0.011 \\
\hline Match availability & 0.086 & 0.005 to 0.168 & 0.037 & 0.085 & 0.008 to 0.163 & 0.031 \\
\hline \multicolumn{7}{|c|}{ Points per league match $\uparrow$} \\
\hline Injury incidence & -0.026 & -0.048 to -0.003 & 0.026 & -0.024 & -0.046 to -0.002 & 0.035 \\
\hline Injury burden & -0.002 & -0.003 to -0.001 & $<0.001$ & -0.002 & -0.003 to -0.001 & $<0.001$ \\
\hline Match availability & 0.019 & 0.009 to 0.029 & $<0.001$ & 0.019 & 0.009 to 0.028 & $<0.001$ \\
\hline \multicolumn{7}{|l|}{ UEFA SCC $¥$} \\
\hline Injury incidence & 0.228 & -0.533 to 0.990 & 0.557 & 0.198 & -0.634 to 1.030 & 0.641 \\
\hline Injury burden & -0.016 & -0.032 to 0.001 & 0.062 & -0.021 & -0.042 to -0.001 & 0.043 \\
\hline Match availability & 0.179 & -0.009 to 0.367 & 0.062 & 0.205 & 0.002 to 0.408 & 0.048 \\
\hline
\end{tabular}

The findings in the present study are in line with those in previous studies of the elite leagues in Qatar ${ }^{9}$ and Iceland, ${ }^{7}$ while no correlation between the team's final league ranking and season injury incidence was observed in another study conducted on a French professional team over 15 seasons. ${ }^{8}$ The exact reason why low injury rates increase a team's chances of success has yet to be established, but there are several plausible explanations. For instance, high match availability means that the coach can select the best possible players in matches, injuries during a match could affect the results in that match negatively, ${ }^{4}$ and injuries could have negative psychological effects for individual players as well as a whole squad.

The team performance measures for domestic competitions used in the present study are similar to those used in previous studies both for final league ranking ${ }^{7-9}$ and league points. ${ }^{9}$ Other measures of team performance in the league were available, for example, number of matches won, number of goals scored and goal difference, ${ }^{9}$ but it was decided a priori not to include these in the present study to reduce the number of statistical analyses.

Injury incidence is a measure commonly used in the sports epidemiological literature, ${ }^{12} 13$ accounting for the frequency of injuries in relation to the amount of sports exposure. It was also used in two previous studies evaluating injuries and performance in football. ${ }^{8}$ The measure does not, however, account for injury severity, that is, the amount of absence the injury causes from sports participation. For this reason, injury burden was included as it accounts for both the frequency (incidence) and severity (mean absence) of injuries, ${ }^{11}$ and probably gives a better picture of the consequences that injuries will have in a team. The injury burden measure is related to the variable total days lost from injury, as used in two previous studies, ${ }^{79}$ but it also accounts for variations in the amount of football exposure. Finally, match availability was included as an injury rate measure in the present study as it was thought to represent the true impact that injuries could have on a team's performance in matches, and it was associated with all three performance measures.

The result of the present study suggests that injuries that cause a high injury burden, and consequently result in more missed matches and decreased match availability, are more likely to impact negatively on team performance. An 11-year follow-up of the same UCL cohort showed that muscle/tendon injuries to the hamstrings and groin, and ligament sprains and joint injuries to the knee and ankle, account for a majority of the injury burden. ${ }^{11}$ Thus, to reduce the overall injury burden and thereby increase performance, these injury types should be targeted with preventive strategies. Studies have shown that it is indeed possible to prevent hamstring injuries, ${ }^{15} 16$ recurrent ankle sprains ${ }^{17-19}$ and knee joint sprains, particularly ACL injuries, ${ }^{20} 21$ in football. Few of these, however, have shown preventive effects at the highest professional level. ${ }^{15}$

\section{Methodological considerations}

Compared with previous similar studies, a slightly different approach was taken to study the association between injury rates and team performance. Owing to differences between various European first leagues (eg, number of league matches, number of teams qualifying for the UCL and EL tournaments, etc) and between teams (eg, club finances, available players, coaches, etc), it was not feasible to study the direct association between injury rates and team performance measures across several teams, as was done previously in studies including teams from only one country. ${ }^{7-9}$ By using each team's preceding season performance and injury rates for comparison, the influence of interteam and interleague variations on the association between outcomes was removed. Similarly, the variable total points in the league, as recently used in a previous study, ${ }^{9}$ were converted to points per league match, since the number of league matches varied both between countries and between seasons. These slight methodological differences make direct comparisons with previous studies difficult, but the main findings were still consistent with previous research.

In the analyses with European cup performances as the outcome, each team's season performance in the domestic league was used to adjust the analyses, and vice versa. This was based on the hypothesis that a team's performance in the domestic league might influence the performance in European cups, and the other way around. For instance, a team that competes in the UCL or EL tournament, and has no chance of 
winning the league at a late stage of the season, might put more effort into the final UCL or EL matches and, possibly, less effort into the remaining matches in the league. Consequently, it is a study limitation that domestic cup matches were not included in the analyses, since the results of these matches may also influence a team's performance in the league play and in European cups. UEFA SCC is not solely based on a team's season performance in European cups, as it also includes 20\% national association ranking, which is a shortcoming with this measure. Still, it was believed to be a feasible and objective measure of a team's international success, as a complementary measure to the domestic league performance, and the results also followed the same pattern as for domestic performance.

Finally, it is acknowledged that several factors not being considered in this study could have changed between seasons and influenced a team's performance in specific matches or over the season, for example, training regimes, recovery strategies, and match load, ${ }^{22}$ match tactics, match locations and quality of the opposition, ${ }^{24}$ squad rosters, etc, and the influence of such factors on the study results is unknown.

In conclusion, this study showed that injuries had a significant influence on performance in the league play and in European cups in male professional football. The findings stress the importance of injury prevention to increase a team's chances of success.

\section{What are the new findings?}

- Professional football teams with lower season injury rates had better performance in the domestic league play in top European leagues.

- Low season injury rates were also associated with improved performance in international European cups.

- Injuries that cause a high injury burden, that is, muscle/ tendon injuries to the hamstrings and groin, and ligament sprains and joint injuries to the knee and ankle, are more likely to impact negatively on team performance.

\section{How might it impact on clinical practice?}

- The association between injuries and performance is probably one of the most important messages to convey to technical and coaching staff, as well as to other stakeholders in professional sports clubs, in order to continue to improve medical services for the players and to increase efforts to prevent injuries.

Acknowledgements The authors would like to thank the participating teams (coaching and technical staff, medical teams and players) for their participation in the study.

Contributors $\mathrm{MH}, \mathrm{MW}$ and JE were responsible for the conception and design of the study. All authors have been involved in the data collection over the study period. $\mathrm{MH}$ and $\mathrm{HM}$ conducted the analyses which were planned and checked with the coauthors. All authors contributed to the interpretation of the findings and had full access to all data. MH wrote the first draft of the paper, which was critically revised by MW, HM, KK, HB and JE. The final manuscript has been approved by all authors. $\mathrm{MH}$ is the study guarantor.
Funding This study was funded by grants from the Union of European Football Associations (UEFA), Swedish National Centre for Research in Sports and Praktikertjänst $A B$.

Competing interests JE is the first vice chairman of the UEFA Medical Committee.

Ethics approval The study design was approved by the UEFA Medical Committee and the UEFA Football Development Division.

Provenance and peer review Not commissioned; externally reviewed.

\section{REFERENCES}

1 Dvorak J, Junge $A$, Derman $W$, et al. Injuries and illnesses of football players during the 2010 FIFA World Cup. Br J Sports Med 2011;45:626-30.

2 Orhant E, Carling C, Cox A. A three-year prospective study of illness in professional soccer players. Res Sports Med 2010;18:199-204.

3 Parry L, Drust B. Is injury the major cause of elite soccer players being unavailable to train and play during the competitive season? Phys Ther Sport 2006;7:58-64.

4 Ekstrand J, Waldén $M$, Hägglund M. Risk for injury when playing in a national football team. Scand J Med Sci Sports 2004;14:34-8.

5 Hägglund $M$, Waldén $M$, Ekstrand J. UEFA injury study —an injury audit at European Championships 2006 to 2008. Br J Sports Med 2009:43:483-9.

6 Waldén $M$, Hägglund M, Ekstrand J. Football injuries during European Championships 2004-2005. Knee Surg Sports Traumatol Arthrosc 2007:15:1155-62.

7 Árnason Á, Sigurdsson SB, Gudmundsson A, et al. Physical fitness, injuries, and team performance in soccer. Med Sci Sports Exerc 2004:36:278-85.

8 Dauty M, Collon S. Incidence of injuries in French professional soccer players. Int $\mathrm{J}$ Sports Med 2011;32:965-9.

9 Eirale C, Tol JL, Faroog A, et al. Low injury rate strongly correlates with team success in Qatari professional football. Br J Sports Med 2013:47:807-8.

10 Ekstrand J, Hägglund $M$, Waldén M. Injury incidence and injury patterns in professional football: the UEFA Injury Study. Br J Sports Med 2011;45:553-8.

11 Ekstrand J, Hägglund M, Kristenson $\mathrm{K}$, et al. Fewer ligament injuries but no preventive effect on muscle injuries and severe injuries: an 11-year follow-up of the UEFA Champions League injury study. Br J Sports Med 2013;47:732-7.

12 Hägglund $M$, Waldén $M$, Bahr R, et al. Methods for epidemiological study of injuries to professional football players: developing the UEFA model. $\mathrm{Br}$ J Sports Med 2005;39:340-6.

13 Fuller CW, Ekstrand J, Junge $A$, et al. Consensus statement on injury definitions and data collection procedures in studies of football (soccer) injuries. Br J Sports Med 2006:40:193-201.

14 Union of European Football Associations. Club rankings and coefficients. http:/l www.uefa.com/memberassociations/uefarankings/ (accessed 14 Dec 2012).

15 Croisier JL, Ganteaume S, Binet J, et al. Strength imbalances and prevention of hamstring injury in professional soccer players. A prospective study. Am J Sports Med 2008:36:1469-75.

16 Petersen J, Thorborg K, Bachmann Nielsen M, et al. Preventive effect of eccentric training on acute hamstring injuries in men's soccer: a cluster-randomized controlled trial. Am J Sports Med 2011;39:2296-303.

17 Mohammadi F. Comparison of 3 preventive methods to reduce the recurrence of ankle inversion sprains in male soccer players. Am J Sports Med 2007;35:922-6.

18 Surve I, Schwellnus MP, Noakes $T$, et al. A fivefold reduction in the incidence of recurrent ankle sprains in soccer players using the Sport-Stirrup orthosis. Am J Sports Med 1994;22:601-5.

19 Tropp H, Askling C, Gillquist J. Prevention of ankle sprains. Am J Sports Med 1985;13:259-62.

20 Caraffa A, Cerulli G, Projetti M, et al. Prevention of anterior cruciate ligament injuries in soccer. A prospective controlled study of proprioceptive training. Knee Surg Sports Traumatol Arthrosc 1996:4:19-21.

21 Waldén $\mathrm{M}$, Atroshi I, Magnusson $\mathrm{H}$, et al. Prevention of acute knee injuries in adolescent female football players: cluster randomised controlled trial. BMJ 2012;344:e3042.

22 Dupont G, Nedelec M, McCall A, et al. Effect of 2 soccer matches in a week on physical performance and injury rate. Am J Sports Med 2010;38:1752-8.

23 Carling C, Orhant E, LeGall F. Match injuries in professional football: inter-seasonal variation and effects of competition type, match congestion and positional role. Int J Sports Med 2010;31:271-6.

24 Lago C. The influence of match location, quality of opposition, and match status on possession strategies in professional association football. J Sports SCi 2009:27:1463-9. 